\title{
Dynamics of tachyon field in spatially curved FRW universe
}

\author{
Jian-Li Li* and Jian-Pin Wut \\ Center for Relativistic Astrophysics and High Energy Physics, \\ Department of Physics, Nanchang University, 330031, China
}

\begin{abstract}
The dynamics of a tachyon field plus a barotropic fluid is investigated in spatially curved FRW universe. We perform a phase-plane analysis and obtain scaling solutions accompanying with a discussion on their stability. Furthermore, we construct the form of scalar potential which may give rise to stable solutions for spatially open and closed universe separately.
\end{abstract}

*Electronic address: lijianli1985@163.com

$\dagger$ Electronic address: jianpinwu@yahoo.com.cn 


\section{INTRODUCTION}

The nature and the origin of dark energy is a fundamental puzzle in modern cosmology. Most dark energy models can be constructed by using a slowly rolling canonical scalar field, termed as quintessence. However, there has been increasing interests in alternative models with a non-canonical kinetic term. Among these models the most general formalism perhaps is k-essence [1]. A more specific case is the tachyon field [2], which is motivated from string theory. It can be viewed as a special case of k-essence with Dirac-Born-Infeld (DBI) action [3]. Although the tachyon is an unstable field, its state parameter in the equation of state varies smoothly between -1 and 0 , thus many authors have already considered the tachyon field as a suitable candidate for a viable model of dark energy phenomenologically [4-13]. For a review, we can refer to Ref.[14].

However, the dynamical dark energy models driven by a scalar field suffer from the socalled fine-tuning problem and coincidence problem. In order to address these problems, one may employ scalar field models exhibiting scaling solutions [8, 9, 15 34]. The scaling solutions as dynamical attractors can considerably alleviate these two problems. Furthermore, by investigating the nature of scaling solutions, one can determine whether such behavior is stable or just a transient feature and explore the asymptotic behavior of the scalar field potential.

Many authors have investigated a lot of scalar field models containing scaling solutions. For instance, a canonical scalar field with an exponential potential has scaling attractor solutions [16]. For quintessence dark energy model, there are two scaling solutions. One is fluid-scalar field scaling solution, which remains subdominant for most of the cosmic evolution. It is necessary that the scalar field mimics the background energy density (radiation/matter) in order to respect the nucleosynthesis constraint and can also alleviate the fine-tuning problem of initial conditions. The other is scalar field dominated scaling solution, which is a late time attractor and gives rise to the accelerated expansion. Since the fluid-scalar field scaling solution is non-accelerating, we need an additional mechanism exit from the scaling regime so as to enter the scalar field dominated scaling solution at late times. For the discussion on the exiting mechanism, we can refer to Refs. [14, 35 [39].

For tachyon field dark energy, the scaling solutions have also been investigated by many authors, for example Refs.[4 13]. To be considered as a realistic model of dark energy, it is 
found that the fluid-scalar field scaling solutions are absent and only the scalar field dominated scaling solutions exist [6]. This is very different from the quintessence case. Therefore, just as pointed out in [13], tachyon models require more fine-tuning to be consistent with observations. Nevertheless, it is worthwhile to point out that the fluid-scalar field scaling solutions may be obtained when the Gauss-Bonnet coupling between tachyon field and fluid is considered in the tachyon dark energy model[34].

Although the latest results of WMAP5 have placed a constraint, $-0.063<\Omega_{k}<0.017$ [40], on the flatness of our observable universe, indicating that our observable universe is very close to flatness, it is still possible that our observable universe is spatially curved. Therefore, it is also interesting to investigate the dynamical behavior of dark energy models in a spatially curved FRW universe. Recently Copeland et.al. have extended such investigations to the quintessence model in spatially curved FRW universe[41], following the strategy they have

developed in Ref.[28]. Sen and Devi [11] have also explored the scaling solutions with tachyon in modified gravity model employing the same method. In this Letter, we will closely follow this route to investigate the dynamics of tachyon dark energy model in spatially curved universe.

Our Letter is organized as follows. In Section II we present the associated equations of motion for the tachyon field including the background fluid and obtain the scaling solutions. Then, we analyze the stability of these solutions. In Section III we turn to construct the scalar potential leading to such scaling solutions. In particular, its asymptotical forms are obtained in various circumstances for spatially open and closed universe respectively.

\section{DYNAMICS OF THE TACHYON FIELD IN THE PRESENCE OF A FLUID}

Let us start with a cosmological model in which the universe is filled with a tachyon field $\phi$ evolving with a positive potential $V(\phi)$ and a barotropic fluid with an equation of state $p_{\gamma}=(\gamma-1) \rho_{\gamma}$, where $\gamma$ is the adiabatic index. We note that $\gamma=1$ for a pressureless dust and $\gamma=4 / 3$ for radiation. The pressure and the energy densities of the tachyon field $\phi$ are respectively given by

$$
\begin{gathered}
p_{\phi}=-V(\phi) \sqrt{1-\dot{\phi}^{2}}, \\
\rho_{\phi}=\frac{V(\phi)}{\sqrt{1-\dot{\phi}^{2}}},
\end{gathered}
$$


then the effective adiabatic index of the tachyon field is given by

$$
\gamma_{\phi}=\frac{\rho_{\phi}+p_{\phi}}{\rho_{\phi}}=\dot{\phi}^{2}
$$

From the above equation, we can see that $0<\gamma_{\phi}<1$.

As shown in Ref.[11] [28] [41], either in a spatially curved FRW universe or a flat universe but described by modified gravity models, the Friedmann equation of the universe can be uniformly written as an effective form

$$
H^{2}=\frac{\rho}{3} L^{2}(\rho(a))
$$

where we have set $8 \pi / m_{p}=1 . H \equiv \dot{a} / a$ is the Hubble parameter and $\rho(a)$ is the total energy density of the universe. In this paper, we adopt the notion $H=\epsilon \sqrt{H^{2}}$, where $\varepsilon=+1$ for expanding universe and $\varepsilon=-1$ for contracting universe. The function $L(\rho(a))$ is assumed to be positive-definite without loss of generality. We also note that when $L(\rho(a))=1$, the effective Friedmann equation (44) can be reduced to the standard spatially flat case.

The energy conserved equation of matter and tachyon field are respectively

$$
\begin{gathered}
\dot{\rho}_{\gamma}+3 \gamma H \rho_{\gamma}=0, \\
\frac{\ddot{\phi}}{1-\dot{\phi}^{2}}+3 H \dot{\phi}+\frac{V_{, \phi}}{V}=0 .
\end{gathered}
$$

Now we intend to demonstrate a phase-plane analysis on this system. As usual, we define

$$
\begin{gathered}
X=\dot{\phi}, \quad Y \equiv \frac{\sqrt{V(\phi)}}{\sqrt{\rho}}, \\
\lambda \equiv-\frac{V_{, \phi}}{L V^{3 / 2}}, \quad \Gamma \equiv V \frac{V_{, \phi \phi}}{V_{, \phi}^{2}},
\end{gathered}
$$

where $\rho$ is the total energy density of the universe and the subscript $N$ denotes the derivative

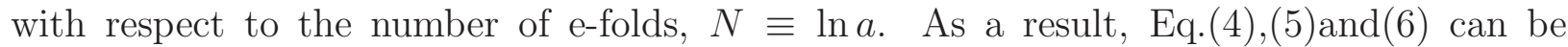
rewritten in the form

$$
\begin{gathered}
X_{, N}=\left(X^{2}-1\right)(3 X-\epsilon \sqrt{3} Y \lambda) \\
Y_{, N}=\frac{Y}{2}\left[-\epsilon \sqrt{3} X Y \lambda+3 \gamma-\frac{3 Y^{2}\left(\gamma-X^{2}\right)}{\sqrt{1-X^{2}}}\right], \\
\lambda_{, N}=-\sqrt{3} \epsilon X Y \lambda^{2}\left(\Gamma-\frac{3}{2}\right)-3 \lambda\left[\frac{\left(\gamma-X^{2}\right) Y^{2}}{\sqrt{1-X^{2}}}-\gamma\right] \rho \frac{\partial \ln L}{\partial \rho},
\end{gathered}
$$


and the constraint equation for the total energy density becomes

$$
\frac{Y^{2}}{\sqrt{1-X^{2}}}+\frac{\rho_{\gamma}}{\rho}=1 .
$$

Since $0 \leq \frac{Y^{2}}{\sqrt{1-X^{2}}} \leq 1$, the allowed range of $X$ and $Y$ is $0 \leq X^{2}+Y^{4} \leq 1$, namely $0 \leq Y^{2} \leq 1$ and $0 \leq X^{2} \leq 1$.

We consider the special case $\lambda=$ const. Taking a derivative with respect to the scalar field $\phi$, then from Eq.(8) we obtain

$$
\Gamma=\frac{3}{2}+\frac{d \ln L}{d \ln V}
$$

The fixed points for this system can be obtained by setting $X_{,_{N}}=0$ and $Y_{,_{N}}=0$ in Eqs. (9) and (10). Essentially we have four fixed points:

$$
\begin{gathered}
X_{c}=0, Y_{c}=0, \\
X_{c}= \pm 1, Y_{c}=0, \\
X_{c}=\frac{\lambda}{\sqrt{3}} Y_{s}, Y_{c}=Y_{s}, \\
X_{c}= \pm \sqrt{\gamma}, Y_{c}= \pm \frac{\sqrt{3 \gamma}}{\lambda},
\end{gathered}
$$

where $Y_{s}$ is defined by

$$
Y_{s}=\sqrt{\frac{\sqrt{\lambda^{4}+36}-\lambda^{2}}{6}} .
$$

We now investigate the stability around the critical points by evaluating the eigenvalues of the matrix $\mathcal{M}$. The way of evaluating eigenvalues has been given in [14] [16] [42]. Here we list our analysis corresponding to each solution as follows and then summarize the results in Table I.

1. For the fluid dominated solution $\left(X_{c}=0, Y_{c}=0\right)$, the eigenvalues are $\mu_{1}=-3, \mu_{2}=$ $3 \gamma / 2$. Therefore, this critical point is an unstable saddle point for $\gamma>0$, whereas it is a stable node for $\gamma=0$ in an expanding universe $(\epsilon=1)$. However, since the fixed point leads to the density parameter $\Omega_{\phi} \equiv \frac{\rho_{\phi}}{\rho}=0$, it cannot be used as a late-time attractor.

2. The fixed points $\left(X_{c}= \pm 1, Y_{c}=0\right)$ correspond to the scalar field kinetic dominated solution with $\gamma_{\phi}=\dot{\phi}^{2}$. The eigenvalues are $\mu_{1}=6, \mu_{2}=3 \gamma / 2$, indicating that this point is unstable in an expanding universe. However, in a contracting universe $(\epsilon=-1)$, it implies unconditional stability when these solutions exist. 
3. For the well-known solution $\left(X_{c}=\lambda Y_{s} / \sqrt{3}, Y_{c}=Y_{s}\right)$, we have eigenvalues $\mu_{1}=$ $-3+\frac{\lambda^{2}}{2} Y_{s}^{2}, \mu_{2}=-3 \gamma+\lambda^{2} Y_{s}^{2}$, where satisfies $-3 \leq \mu_{1}<-3 / 2$. Moreover, we have $\mu_{2} \leq 0$ for $\gamma \geq \gamma_{s} \equiv \frac{\lambda^{2}}{3} Y_{s}^{2}$, indicating that, in an expanding universe, the fixed point is stable for $\gamma \geq \gamma_{s}$, whereas it is an unstable saddle point for $\gamma<\gamma_{s}$. On the contrary, in a contracting universe the critical point is unstable for $\gamma \geq \gamma_{s}$ and be a saddle point for $\gamma<\gamma_{s}$. Since in this solution, the kinetic energy of the tachyon field is proportional to the potential energy, we call this solution as the scalar field dominated scaling solution as Ref.[41].

4. The value $\gamma_{\phi}$ at the last critical points $\left(X_{c}= \pm \sqrt{\gamma}, Y_{c}= \pm \frac{\sqrt{3 \gamma}}{\lambda}\right)$ is $\gamma_{\phi}=\gamma$, which means both energy densities $\rho_{\phi}$ and $\rho_{\gamma}$ decrease with the same rate. The eigenvalues are $\mu_{1,2}=\frac{3}{4}\left[\gamma-2 \pm \sqrt{17 \gamma^{2}-20 \gamma+4+\frac{48}{\lambda^{2}} \gamma^{2} \sqrt{1-\gamma}}\right]$. From Eq.(12) we have $0 \leq \gamma \leq$ $\gamma_{s}=\frac{\lambda^{2}}{3} Y_{s}^{2}$, note that $\gamma_{s}$ is always smaller than 1 . The real parts of $\mu_{1}$ and $\mu_{2}$ are both negative under the condition $\gamma \leq \gamma_{s}$. Obviously, it is an unstable solution in a contracting universe, whereas in an expanding universe the scaling solution is always stable. However, we need to caution that the existence of the scaling solution requires the condition $0 \leq \gamma \leq \gamma_{s}<1$, which is not satisfied for known realistic fluids. So it is always treated as an unpractical solution.

\begin{tabular}{|c|c|c|c|c|c|c|c|c|c|c|c|c|}
\hline & \multicolumn{2}{|c|}{$\begin{array}{l}X=0 \\
Y=0\end{array}$} & \multicolumn{2}{|c|}{$\begin{array}{l}X=1 \\
Y=0\end{array}$} & \multicolumn{2}{|c|}{$\begin{array}{c}X=-1 \\
Y=0\end{array}$} & \multicolumn{2}{|c|}{$\begin{array}{c}X=\frac{\lambda}{\sqrt{3}} Y_{s} \\
Y=Y_{s}\end{array}$} & \multicolumn{2}{|c|}{$\begin{aligned} X & =\sqrt{\gamma} \\
Y & =\frac{\sqrt{3 \gamma}}{\lambda}\end{aligned}$} & \multicolumn{2}{|c|}{$\begin{aligned} X & =-\sqrt{\gamma} \\
Y & =-\frac{\sqrt{3 \gamma}}{\lambda}\end{aligned}$} \\
\hline & exists & stable & exists & stable & exists & stable & exists & stable & exists & stable & exists & stable \\
\hline$\epsilon=+1$ & $\forall \lambda, \forall \gamma$ & No & $\forall \lambda, \forall \gamma$ & No & $\forall \lambda, \forall \gamma$ & No & $\forall \lambda, \forall \gamma$ & $\gamma \geq \gamma_{s}$ & $\begin{array}{c}\lambda>0 \\
0 \leq \gamma \leq \gamma_{s}\end{array}$ & Yes & $\begin{array}{c}\lambda<0 \\
0 \leq \gamma \leq \gamma_{s}\end{array}$ & Yes \\
\hline$\epsilon=-1$ & $\forall \lambda, \forall \gamma$ & No & $\forall \lambda, \forall \gamma$ & $\begin{array}{l}\text { when } \\
\text { exists }\end{array}$ & $\forall \lambda, \forall \gamma$ & $\begin{array}{l}\text { when } \\
\text { exists }\end{array}$ & $\forall \lambda, \forall \gamma$ & No & $\begin{array}{c}\lambda>0 \\
0 \leq \gamma \leq \gamma_{s}\end{array}$ & No & $\begin{array}{c}\lambda<0 \\
0 \leq \gamma \leq \gamma_{s}\end{array}$ & No \\
\hline
\end{tabular}

TABLE I: The existence and stability conditions for an expanding $(\epsilon=1)$ and a contracting $(\epsilon=-1)$ universe, containing a tachyon field and a fluid with the adiabatic index $\gamma$.

In addition, we also plot some representative figures (FIG.1 and FIG.2) for the evolution of universe, from which we can see the better picture of the late time acceleration. From 


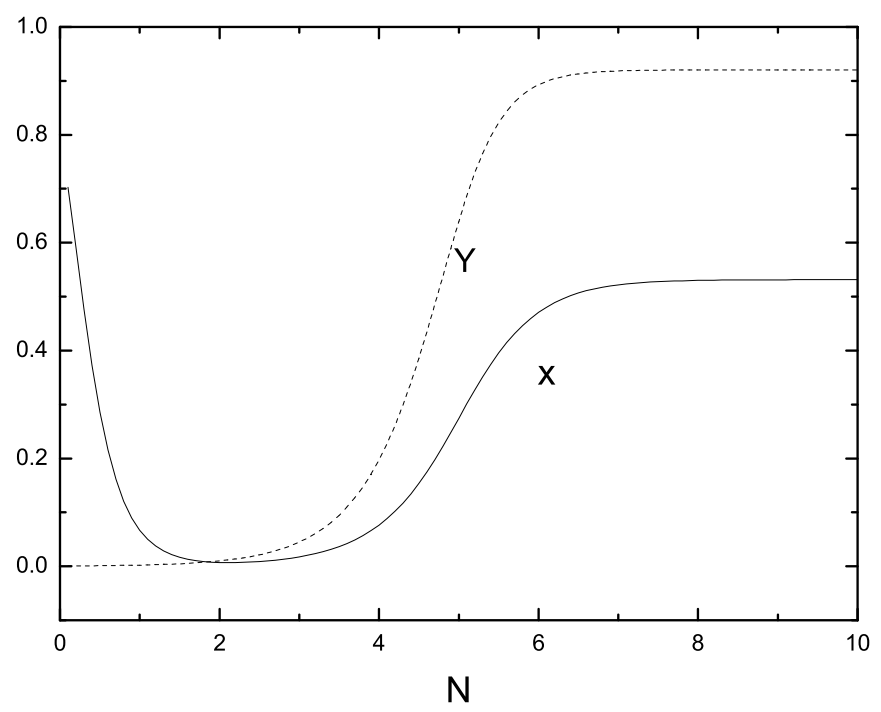

FIG. 1: The evolution of the parameters $x$ and $y$ in the presence of a barotropic fluid with $\gamma=1$. Here we take $\lambda=1$ and the initial conditions $x_{i}=0.8, y_{i}=5.0 \times 10^{-4}$ when $N=0$.

FIG.1, we can see that when $\gamma=1>\gamma_{s}$, the evolution of the parameters $x$ and $y$ approach the critical point $\left(X_{c}=\lambda Y_{s} / \sqrt{3} \simeq 0.53, Y_{c}=Y_{s} \simeq 0.92\right)$ as $N$ increases. From FIG.2, we can see that in the attractor regime, the equation of state $w_{\phi} \simeq-0.72$ and the density parameter $\Omega_{m}=0$ but $\Omega_{\phi}=1$ which is just tachyon field dominated epoch.

\section{TACHYON FIELD POTENTIALS IN CURVED UNIVERSE}

In the rest of our Letter we focus on the scaling solution given by Eq.(16), and construct the tachyon field potential which may give rise to this late time attractor, provided that the specific forms of $L(\rho)$ is given for spatially curved universe. At critical points we always have $Y_{c}=\frac{\sqrt{V}}{\sqrt{\rho}}=$ constant, thus $L(\rho)$ can be described as $L(V)$. Substituting this into Eq. (8), we can obtain

$$
\int \frac{d V}{L V^{3 / 2}}=-\lambda \phi
$$

Therefore, given a specific form of $L$, it is possible to derive the potential for tachyon field in the scaling solution by integrating Eq.(19). 


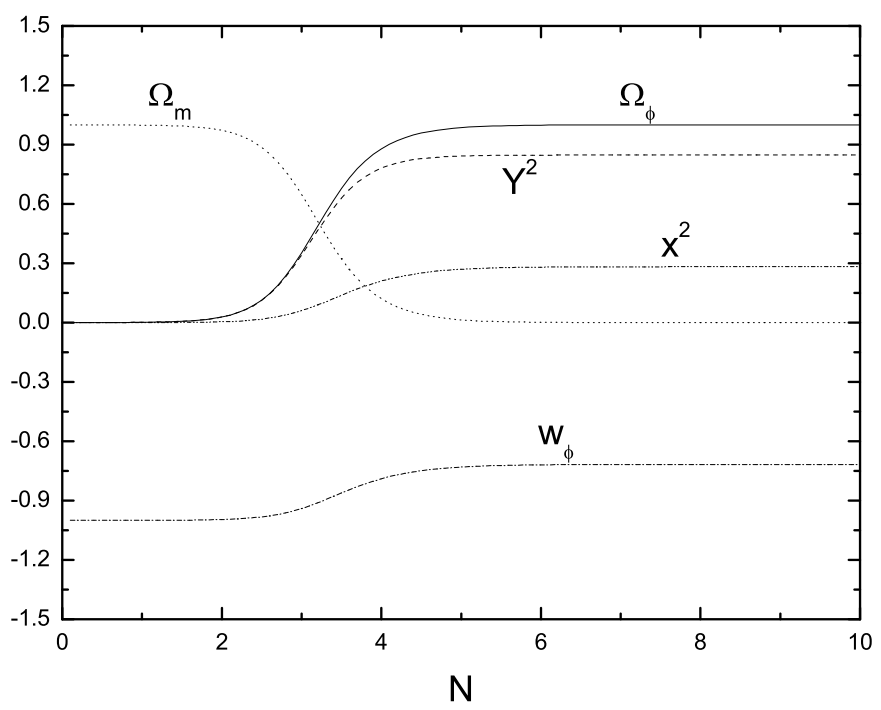

FIG. 2: The evolution of $\Omega_{\phi}, \Omega_{m}, w_{\phi}, x^{2}$ and $y^{2}$ for $\gamma=1$. Here we take $\lambda=1$ and the initial conditions $x_{i}=0.0085, y_{i}=0.0085$ when $N=0$.

\section{A. Open FRW universe}

In open FRW universe, $L(\rho)$ is given by

$$
L(\rho)=\sqrt{1+\frac{3}{\rho a^{2}}},
$$

At first we assume $\lambda \neq 0$ and discuss the form of the potential in an expanding universe. Then, the special case of $\lambda=0$ is presented at the end of this subsection.

case $(\mathbf{i}) \lambda \neq 0$

When the universe is expanding $(\epsilon=1)$, the solution given by Eq.(16) is stable for $\gamma \geq \gamma_{s}=\frac{1}{3} \lambda^{2} Y_{s}^{2}$. By using the energy conserved equation of tachyon field

$$
\dot{\rho_{\phi}}+3 \frac{\dot{a}}{a} \gamma_{\phi} \rho_{\phi}=0
$$

Therefore, we can obtain

$$
a=a_{(i)} \rho_{\phi}^{\frac{1}{3 \gamma_{(i) \phi}}} \rho_{\phi}^{-\frac{1}{3 \gamma_{\phi}}}
$$

Because this solution is the scalar field dominated scaling solution, the fluid is absent. 
Therefore, we can have

$$
a=a_{(i)} \rho^{\frac{1}{3 \gamma(i)}} \rho^{-\frac{1}{3 \gamma}}
$$

With the help of the expression of the effective adiabatic index $\gamma_{\phi}=X_{c}^{2}=\frac{1}{3} \lambda^{2} Y_{s}^{2}$, the scale factor $a$ is given by

$$
a=A \rho^{-\frac{1}{\lambda^{2} Y_{s}^{2}}}, A=a_{(i)} \rho_{(i)}^{\frac{1}{\lambda^{2} Y_{s}^{2}}}
$$

where the subscript $(i)$ refers to some initial time.

The correction function $L$ given by Eq.(20) is then rewritten as

$$
L(\rho)=\sqrt{1+\frac{3}{A^{2}} \rho^{\mu}}
$$

where $\mu=\frac{2}{\lambda^{2} Y_{s}^{2}}-1$, and for the region of existence of these solutions, the valid range of $\mu$ is $-1 / 3<\mu<\infty$.

At the critical point, $Y_{c}$ is a constant. Substituting $Y_{s}=\sqrt{V / \rho}$ into Eq. (19), the scaling solution potential will correspond to

$$
\int \frac{d V}{V^{3 / 2} \sqrt{1+\frac{3}{A^{2}} Y_{s}^{-2 \mu} V^{\mu}}}=-\lambda \phi .
$$

Subsequently, we will classify the asymptotic behavior of the scalar field potential above in terms of the sign of the parameter $\mu$. In addition, we also note that the equation (26) is invariant under the transformation $\lambda \rightarrow-\lambda$ and $\phi \rightarrow-\phi$. Therefore, our work can be restricted to the first quadrant without loss of generality.

1. For $\mu<0$ (i.e. $\lambda^{2} Y_{s}^{2}>2$ ), in an expanding universe at early times, where the curvature is negligible, the potential for the tachyon field is obtained as $V \sim 4 \lambda^{-2} \phi^{-2}$. However, at late times, the universe becomes one dominated by the curvature, the asymptotic form of the potential is a function like $V \propto \lambda^{-\frac{2}{\mu+1}} \phi^{-\frac{2}{\mu+1}}$.

2. For $\mu>0$ (i.e. $\lambda^{2} Y_{s}^{2}<2$ ), we find that at early times, the potential relating to the curvature dominated universe can be approximated by $V \propto \lambda^{-\frac{2}{\mu+1}} \phi^{-\frac{2}{\mu+1}}$. Once the curvature is negligible at late times, it can be shown that the potential is $V \sim 4 \lambda^{-2} \phi^{-2}$.

3. For the special case of $\mu=0$, the correction function $L$ becomes a constant, and it can be seen as a modified Newton's gravitational constant in Eq.(4). The evolving 
background corresponds to a flat Friedmann universe, and the potential asymptotically

has a form of the late-time attractor $V \sim \frac{2 \sqrt{3}}{3} \phi^{-2}$. Note that in this special case, the scalar field and the curvature scale together.

We summarize above results in the Case A category of Table II.

However, according to the results of Table I, we know that in a contracting universe, the scalar field dominated solution is not stable, but the kinetic dominated solution is stable. We must point out that in kinetic dominated solution, since $Y_{c}=0$, we have the potential $V(\phi)=0$.

- case (ii) $\lambda \approx 0$

For the special case of $\lambda=0$, the potential will be a constant. The solution described by Eq.(16) is still applicable and from it we obtain $X_{c}=0, Y_{c}=1$, which is nothing but a de-sitter solution, as substituting this into Eqs.(1)and (2) we have $\dot{\phi}=0$ and the state parameter $\omega=-1$. The exact solution can also be obtained by substituting these into the Eqs.(44), (5) and (6).

From Table I, we can conclude that the tachyon field dominated solution is stable in the expanding universe, but unstable in a contracting universe.

\section{B. Closed FRW universe}

Now we turn to obtain the tachyon potential at the stable critical point for a closed FRW universe. In this case $L(\rho(a))$ is given by

$$
L(\rho)=\sqrt{1-\frac{3}{\rho a^{2}}} .
$$

Obviously, the valid range of the correction function is $1 \geq L \geq 0$. Specially when $L=0$, the scale factor is a constant, which neither expanding nor contracting. Therefore, we can assume at that point, the universe probably experiences a bounce and the evolution transmits from expanding(contracting) to contracting (expanding). Let us follow this idea to do the rest of the analysis. As before, we begin our discussion on the expanding universe and then analyze the difference between expanding and contracting universe for the scaling solution.

$\boldsymbol{\nabla}$ case (i) $\lambda \neq 0$ 
In an expanding universe $(\epsilon=1)$, the scaling solution given by Eq.(16) exists for $\gamma \geq$ $\gamma_{s}=\frac{\lambda^{2} Y_{s}^{2}}{3}$, and the scale factor is still given by Eq.(24). Then we can rewrite the correction function as

$$
L(\rho)=\sqrt{1-\frac{3}{A^{2}} \rho^{\mu}}
$$

Subsequently, using $Y_{c}=\frac{\sqrt{V}}{\sqrt{\rho}}$ and substituting (28) into Eq.(19), we obtain

$$
\int \frac{d V}{V^{3 / 2} \sqrt{1-\frac{3}{A^{2}} Y_{s}^{-2 \mu} V^{\mu}}}=-\lambda \phi .
$$

As the case in the open FRW universe, we discuss the asymptotic behavior of the scaling potential by classifying the valid range of $\mu$ into positive, negative and vanishing regions. We can also choose to work in the first quadrant without loss of generality. These are summarized in Case B of Table II.

1. For $\mu>0$ (i.e. $\lambda^{2} Y_{s}^{2}<2$ ), at early time of an expanding universe, we have $a^{-\lambda^{2} Y_{s}^{2}}<a^{-2}$. However, the universe cannot be dominated by the curvature as the correction function is bounded in a closed universe, otherwise the right-hand side of the Friedmann equation (4) would be negative. Consider a pragmatic point, the curvature is just subdominant to the scalar field. When $L(\rho) \rightarrow 0$, the total energy density has a maximum $\rho \rightarrow \rho_{\max } \equiv\left(\frac{A^{2}}{3}\right)^{1 / \mu}$, and the universe has a constant scale factor. From Eq.(17), we conclude that the potential is almost a constant with $V \approx Y_{c}^{2}\left(\frac{A^{2}}{3}\right)^{1 / \mu}$. Subsequently, the universe starts to expand, the tachyon field gradually become dominated over the curvature. At last, one can neglect the contribution of the curvature. Therefore, the universe can be considered a flat FRW spacetime, and the asymptotic form of the potential become $V \sim 4 \lambda^{-2} \phi^{-2}$.

2. For $\mu<0$ (i.e. $\lambda^{2} Y_{s}^{2}>2$ ), the universe dominated by the tachyon field starts to expand from an approximate flat FRW spacetime, and the scaling solution potential has the form $V \sim 4 \lambda^{-2} \phi^{-2}$. As time goes on, the total energy density will reach the maximal value, $\rho \rightarrow \rho_{\max } \equiv\left(\frac{A^{2}}{3}\right)^{1 / \mu}$, where the contribution of the curvature and the scalar field are almost equal. The scale factor does not change as $H \rightarrow 0$, and the potential is almost a constant $V \approx Y_{c}^{2}\left(\frac{A^{2}}{3}\right)^{1 / \mu}$. After this turning point, the universe changes from expanding to collapsing. From Table I, we see that in a contracting universe, the late time scalar field scaling solutions are unconditionally unstable. Once the universe 
starts to collapse, the solution will asymptote towards the kinetic dominated solution with $\gamma_{\phi}=1$.

3. For $\mu=0$, the situation is similar to the open universe scenario. The correction function $L$ becomes a constant. The potential has the form $V \sim \frac{2 \sqrt{3}}{3} \phi^{-2}$, where the contributions of the scalar field and curvature to the Friedmann equation scale together.

As mentioned in the subsection for open universe, for a contracting universe if the kinetic dominated solution exists they will be unconditionally stable, and the potential $V(\phi)=0$.

$\boldsymbol{\nabla}$ case $(\mathrm{ii}) \lambda \approx 0$

Similar to our description of an open universe scenario, this case corresponds to a constant potential, and only the fixed point described by (16) exists. For $\lambda=0$, which results in an expanding universe, the attractor solution reduces to $X_{c}=0$ and $Y_{c}=1$, describing an exact de-Sitter solution. We notice that the assumption of taking $\phi$ as a monotonically varying function of time breaks down if $X_{c} \propto \dot{\phi}=0$.

Furthermore, based on our previous analysis it is worth noting that when $\lambda \approx 0$, the critical point (16) is unstable in a contracting universe, but becomes a stable one in an open universe.

Finally, from the above analysis, we note that the evolution of the universe including the curvature term is same as the case without the curvature term [6]. However, due to the curvature term, the required potential is different. As shown in [6], in the case without curvature term, the required potential is always $V(\phi) \sim \phi^{-2}$. But when the curvature term is taken into account, the potential has various form in the different epochs of the universe.

\section{CONCLUSION AND DISCUSSION}

In this Letter we have investigated the dynamics of a tachyon field in FRW universe with spatial curvature. Following the scheme presented in [28, 41], we denoted the modification of the cosmological equation due to the spatial curvature by a general function $L(\rho)$, and then derived the conditions under which the system can enter a scaling solution. In particular, we obtain an attractor solution, where the tachyon field dominates over the fluid and the kinetic 


\begin{tabular}{|c|c|c|c|c|c|c|}
\hline & \multicolumn{3}{|c|}{ Case $A$} & \multicolumn{3}{c|}{ Case B } \\
\hline & $\mu<0$ & $\mu=0$ & $\mu>0$ & $\mu<0$ & $\mu=0$ & $\mu>0$ \\
\hline \hline \multirow{2}{*}{ Early times } & $V \sim \phi^{-2}$ & $V \sim \phi^{-2}$ & $V \sim \phi^{-\frac{2}{\mu+1}}$ & $V \sim \phi^{-2}$ & $V \sim \phi^{-2}$ & $V \sim Y_{c}^{2}\left(\frac{A^{2}}{3}\right)^{1 / \mu}$ \\
& negligible & scaling & curvature & negligible & scaling & $\begin{array}{c}\text { curvature } \\
\text { only just }\end{array}$ \\
& curvature & curvature & dominated & curvature & curvature & $\begin{array}{c}\text { subdominant } \\
\text { Late times }\end{array}$ \\
& $V \sim \phi^{-\frac{2}{\mu+1}}$ & $V \sim \phi^{-2}$ & $V \sim \phi^{-2}$ & $V \sim Y_{c}^{2}\left(\frac{A^{2}}{3}\right)^{1 / \mu}$ & $V \sim \phi^{-2}$ & $V \sim \phi^{-2}$ \\
& curvature & scaling & negligible & curvature & scaling & negligible \\
& dominated & curvature & curvature & only just & curvature & curvature \\
& & & subdominant & & \\
\hline
\end{tabular}

TABLE II: This table summarizes the asymptotic behavior of an expanding $(\epsilon=1)$ universe described by the scaling solutions. Case $A$ refers to open FRW universe, and Case $B$ corresponds to closed FRW universe.

energy of the field scales with its potential energy. Furthermore, given the modification function for an open and closed universe respectively, we discussed the form of the scalar potential which gives rise to the late time attractor solution. For spatially open universe, we conclude that in regions where the curvature is negligible, the asymptotic form of the potential is $V \propto \phi^{-2}$, while in regions where it is dominant, the approximated potential will be $V \propto \phi^{-\frac{2}{\mu+1}}$. As far as the spatially closed universe is concerned, we find that the curvature is not allowed to be dominant among the total energy density of the universe, but only subdominant to the scalar field density, where the potential is a constant as $V \approx Y_{c}^{2}\left(\frac{A^{2}}{3}\right)^{1 / \mu}$. When the curvature becomes negligible, the potential can be approximated by $V \sim \phi^{-2}$. Moreover, it was noticed that in a contracting closed universe, the scalar dominated solutions are not stable, and the kinetic dominated solution will be a late time attractor if it exists.

Comparing with the quintessence scalar field dark energy model with spatial curvature [41], the fluid-scalar field scaling solutions is absent in the tachyon dark energy model. In addition, in the quintessence scalar field dark energy model, there are the two forms of the potential: the exponential potential and the power-law potential. But in the tachyon dark energy model, only the power-law potential is required.

In this Letter we only consider the case that the scalar field and the fluid do not interact 
with each other. It is a very interesting question if such an interaction term can be introduced in order to obtain a fluid-tachyon field scaling solutions in curved FRW universe. Effort has been made in present framework, unfortunately, the answer seems to be negative. However,

it should be very desirable if we consider adding a Gauss-Bonnet coupling as in [34]. We expect to make further progress along this direction.

\section{Acknowledgement}

We are grateful to Prof. Yi Ling and Da-Zhu Ma for helpful discussions. This work is partly supported by NSFC(Nos.10663001,10875057), JiangXi SF(Nos. 0612036, 0612038), Fok Ying Tung Eduaction Foundation(No. 111008) and the key project of Chinese Ministry of Education(No.208072). We also acknowledge the support by the Program for Innovative Research Team of Nanchang University.

[1] C. Armendariz-Picon, V. F. Mukhanov, and P. J. Steinhardt, Phys. Rev. Lett. 85, 4438 (2000) arXiv:astro-ph/0004134].

[2] G. W. Gibbons, Phys. Lett. B 537, 1 (2002) arXiv:hep-th/0204008.

[3] L. P. Chimento, Phys. Rev. D 69, 123517 (2004) arXiv:astro-ph/0311613.

[4] T. Padmanabhan and T. R. Choudhury, Phys. Rev. D $66 \quad$ (2002) 081301 arXiv:hep-th/0205055.

[5] F. Piazza and S. Tsujikawa, JCAP 0407 (2004) 004 arXiv:hep-th/0405054.

[6] E. J. Copeland, M. R. Garousi, M. Sami and S. Tsujikawa, Phys. Rev. D 71 (2005) 043003 arXiv:hep-th/0411192.

[7] B. Gumjudpai, T. Naskar and J. Ward, JCAP 0611 (2006) 006 arXiv:hep-ph/0603210.

[8] S. Tsujikawa, Phys. Rev. D 73 (2006) 103504 arXiv:hep-th/0601178.

[9] Y. Gong, A. Wang and Y. Z. Zhang, Phys. Lett. B 636 (2006) 286 arXiv:gr-qc/0603050].

[10] J. Martin and M. Yamaguchi, Phys. Rev. D 77 (2008) 123508 [arXiv:0801.3375v1].

[11] A. A. Sen and N. C. Devi, Phys. Lett. B 668, 182 (2008) arXiv:0804.2775v2].

[12] B. Gumjudpai and J. Ward, arXiv:0904.0472v1 ].

[13] G. Calcagni, A.R. Liddle ,Phys.Rev. D74 (2006) 043528 arXiv:astro-ph/0606003. 
[14] E. J. Copeland, M. Sami, S. Tsujikawa ， Int.J.Mod.Phys.D15:1753-1936,2006 arXiv:hep-th/0603057].

[15] P. G. Ferreira and M. Joyce, Phys.Rev. D58 (1998) 023503 arXiv:astro-ph/9711102 v3].

[16] E. J. Copeland, A. R. Liddle and D. Wands, Phys. Rev. D 57, 4686 (1998) arXiv:gr-qc/9711068.

[17] A. P. Billyard, A. A. Coley and R. J. van den Hoogen, Phys.Rev. D58 (1998) 123501 arXiv:gr-qc/9805085.

[18] A. R. Liddle and R. J. Scherrer, Phys.Rev. D59 (1998) 023509 [ arXiv:astro-ph/9809272 ].

[19] J. P. Uzan, Phys. Phys.Rev. D59 (1999) 123510 arXiv:gr-qc/9903004].

[20] R. J.van den Hoogen, A. A. Coley and D. Wands, Class.Quantum.Grav.16:1843-1851,1999 [ arXiv:gr-qc/9901014]

[21] A. Nunes and J. P. Mimoso, Phys. Lett. B 488, 423(2000).

[22] A. de la Macorra and G. Piccinelli, Phys.Rev. D61 (2000) 123503 arXiv:hep-ph/9909459 ].

[23] S. C. C. Ng, N. J. Nunes and F. Rosati, Phys.Rev.D64:083510,2001 arXiv:astro-ph/0107321].

[24] C. Rubano and J. D. Barrow, Phys.Rev. D64 (2001) 127301 arXiv:gr-qc/0105037]

[25] Z. K. Guo, Y. S. Piao, R. G. Cai and Y. Z. Zhang, Phys.Lett. B 576, 12 (2003).

[26] Z. K. Guo, Y. S. Piao and Y. Z. Zhang, Phys.Lett. B 568, 1 (2003).

[27] S. Mizuno, S. J. Lee and E. J. Copeland, Phys.Rev. D70 (2004) 043525[ arXiv:astro-ph/0405490.

[28] E. J. Copeland, S. J. Lee, J. E.Lidsey and S. Mizuno, Phys.Rev. D71 (2005) 023526 arXiv:astro-ph/0410110].

[29] M. Sami, N.Savchenko and A. Toporensky, Phys.Rev. D64 (2001) 123526 arXiv:hep-ph/0104186.

[30] V.Pettorino, C. Baccigalupi and F. Perrotta, JCAP 0512 (2005) 003 arXiv:astro-ph/0508586 ].

[31] L.Amendola, S.Tsujikawa and M.Sami, Phys.Lett. B632 (2006) 155-158 [ arXiv:astro-ph/0506222.

[32] S. Tsujikawa and M. Sami,Phys.Lett.B603:113-123,2004 arXiv:hep-th/0409212

[33] L. Amendola, M. Quartin, S. Tsujikawa and I. Waga, Phys.Rev. D74 (2006) 023525 arXiv:astro-ph/0605488.

[34] S. Tsujikawa, M. Sami, JCAP0701:006,2007 arXiv:hep-th/0608178v2] 
[35] T. Barreiro, E. J. Copeland and N. J. Nunes, Phys. Rev. D 61, 127301 (2000).

[36] V. Sahni and L. M. Wang, Phys. Rev. D 62, 103517 (2000).

[37] A. Albrecht and C. Skordis, Phys. Rew. Lett, 84, 2076 (2000).

[38] A. A. Coley and R. J. van den Hoogen, Phys. Rev. D 62, 023517 (2000).

[39] S.A. Kim, A. R. Liddle and S. Tsujikawa, Phys. Rev. D 72, 043506 (2005).

[40] E. Komatsu et al. [WMAP Collaboration], Astrophys. J. Suppl. 180, 330 (2009) arXiv:0803.0547 2 ].

[41] E.J.Copeland, S.Mizuno, M.Shaeri, Phys. Rev. D 79, 103515 (2009) arXiv:0904.0877v2 ].

[42] B. Gumjudpai, T. Naskar, M. Sami, S. Tsujikawa, JCAP 0506:007,2005 arXiv:hep-th/0502191 ]. 